\title{
Triptolide protects podocytes via autophagy in immunoglobulin A nephropathy
}

\author{
SHIKAI LIANG, JUAN JIN, XIAOGANG SHEN, XINXIN JIANG, YIWEN LI and QIANG HE \\ Department of Nephrology, Zhejiang Provincial People's Hospital, Hangzhou, Zhejiang 310014, P.R. China
}

Received December 13, 2017; Accepted July 2, 2018

DOI: $10.3892 /$ etm.2018.6480

\begin{abstract}
Triptolide is often used to treat patients with immunoglobulin A nephropathy (IgAN), especially in Asia. However, its detailed mechanism remains unclear. In vitro experiments were conducted with podocytes exposed to aggregated IgA (aIgA)-MSC1097-conditioned media. A total of four groups were compared in this study: A control group (CON); a healthy supernatant group (HEAs); an IgAN supernatant group (IgANs); and a triptolide group (TRI). First, aggregated IgA1 (aIgA1) was generated by heating monomeric IgA1 (mIgA1) from IgAN patients or healthy subjects. Next, the conditioned supernatant of MSC-1097 cells cultured with aIgA1 (100 mg/l) from IgAN patients (IgANs) or healthy subjects (HEAs) or without aIgA1 (CON) were harvested and used to incubate MPC5 cells. MPC5 cells in the TRI group were cultured with triptolide $(10 \mathrm{ng} / \mathrm{ml})$ and conditioned media from MSC-1097 cells cultured with aIgA1 from IgAN patients. After $24 \mathrm{~h}$ of treatment, MPC5 cells were collected to measure autophagy-related protein levels, including microtubule-associated protein light chain 3 (LC3), p62, cluster of differentiation (CD)63, phosphorylated-protein kinase B (Akt), Akt, p-mammalian target of rapamycin (mTOR), and mTOR, via western blotting, immunofluorescence or both, and to determine apoptosis by flow cytometry. All the results showed no difference between the CON and the HEAs. Compared to the CON and the HEAs, MPC5 cells in the IgANs group showed reduced autophagy, which was presented as decreased levels of LC3-II and CD63, as well as accumulation of p62, and an increased podocyte apoptosis rate. This was partly rescued by the addition of triptolide. Moreover, the $\mathrm{p}$-mTOR/mTOR ratio increased in the IgANs group and decreased in the TRI group. Therefore, these results suggest that triptolide protects podocyte autophagy in $\operatorname{IgAN}$ patients.
\end{abstract}

Correspondence to: Professor Qiang He, Department of Nephrology, Zhejiang Provincial People's Hospital, 158 Shangtang Road, Hangzhou, Zhejiang 310014, P.R. China

E-mail: qianghe1973@126.com

Key words: triptolide, autophagy, podocyte, immunoglobulin A, nephropathy

\section{Introduction}

Immunoglobulin A nephropathy (IgAN) was first described and named by two French scholars, Berger and Hinglais, in 1968 (1). IgAN is typically characterized by IgA deposits in the mesangial area of the glomeruli. Glomerular IgA in biopsy specimens from IgAN patients almost always belong to the IgA1 subclass and are generally polymeric. These abnormal IgA1 peptides show a defect in the galactose molecule. Levels of defective glycosylated Ig A1 are higher in the blood of IgAN patients in comparison to normal individuals (2). Recently, defective IgA1 has been considered to be an important factor for the development and progression of IgAN. It has been reported that (3) Fas mRNA expression and podocyte apoptosis increased markedly upon exposure to conditioned media produced by cultured mesangial cells incubated with aggregated IgA1 ( $\operatorname{Ig} \mathrm{A} 1$ ) from IgAN patients. Both aIgA1 from IgAN patients and the conditioned media from mesangial cells incubated with aIgA1 from IgAN patients inhibited the expression of podocyte nephrin, which is a necessary protein for the proper functioning of the renal filtration barrier (4). The IgA1-immune complex in IgAN patients may increase the production of chemokine (C-X-C motif) ligand 1 (CXCL1) and transforming growth factor-beta (TGF- $\beta$ ) from mesangial cells (5). In turn, CXCL1 and TGF- $\beta$ may exert a synergistic effect on podocytes, inducing podocyte dysfunction and even death. Thus, it can be concluded that the podocyte plays an important role in IgAN. Clinical findings have also consolidated this point of view. Podocytes were found to detach from the glomerular basal membrane (6) and were detected in the urine of IgAN patients. Most importantly, the amount of podocytopenia has been found to be closely associated with the severity of glomerular pathological lesions (7). Autophagy is especially important for the maintenance of podocytes, which have only a limited capacity for regeneration (8). For example, aging mice with podocyte-specific deletion of autophagy-related genes exhibited proteinuria and glomerulopathy (9).

Triptolide is a common Chinese herb often used as an immunosuppressant in glomerulonephritis (10). However, its exact mechanism of action remains unclear. The present study was designed to explore the renal protective effect of triptolide on podocyte autophagy upon treatment with the conditioned media from mesangial cells incubated with aIgA1 from IgAN patients. 
CD63, also known as lysosomal-associated membrane protein-3, is a widely used marker of secretory lysosomes, and positively correlates with the formation of autolysosomes. As a soluble protein in mammalian cells, microtubule-associated protein light chain 3 (LC3) exists in the two forms of LC3-I and LC3-II. LC3-II, the conjugated product of LC3-I and phosphatidyl ethanolamine (PE), is a necessary molecule during the formation of autophagosome. Hence an elevated LC3-II or LC3-II/LC3-I ratio is considered as the typical and standard marker for activation of autophagy. At present, the PI3K/Akt signalling pathway has been shown to be involved in multiple physiological and pathological processes and is one of the most important pathways regulating autophagy. The phosphorylation of mTOR, a major suppressor of autophagy, can be promoted by phosphorylated Akt (p-Akt). Therefore, the above were selected as the autophagic markers of reference in our study.

\section{Materials and methods}

Cell culture and grouping. We used a conditionally immortalized mouse podocyte cell line (MPC5, Tong-Pai Bio-tech Co., Ltd., Shanghai, China). The aIgA1 purification protocol has been described in detail previously (11). Of the 35 patients in this study, who were admitted to Zhejiang Provincial Peope's Hospital (Hangzhou, China) from January 1, 2013 to December 31, 2014, 18 were male and 17 were female. Five healthy male subjects were also enrolled in this study. The study has obtained human research ethics approval from the ethics committee of Zhejiang Provincial People's Hospital and all subjects provided written consent (KY2014011).

All experiments were performed on podocytes differentiated for 10-14 passages. MPC5 cells were exposed to media conditioned by mouse mesangial cells (MSC1097, Tong-Pai Bio-tech Co., Ltd.), which had been cultured for $24 \mathrm{~h}$ without additional treatment (control group, $\mathrm{CON}$ ) or in the presence of the following: 1) aIgA1 (100 mg/l) from IgAN patients (IgANs group); or 2) aIgA1 (100 mg/l) from healthy subjects (HEAs group); 3) triptolide (10 ng/ml; Sigma-Aldrich; Merck KGaA, Darmstadt, Germany) and aIgA1 (100 mg/l) from IgAN patients (TRI group), in RPMI 1640 medium. After co-culture for $24 \mathrm{~h}$, cells were collected for further experiments. We selected $100 \mathrm{mg} / \mathrm{l}$ aIgAland $10 \mathrm{ng} / \mathrm{ml}$ triptolide to treat the cells just according to previous researches $(12,13)$.

Western blot analysis. Podocytes were lysed in a lysis buffer at $4^{\circ} \mathrm{C}$. After being centrifuged at $12,000 \mathrm{rpm}$ for $5 \mathrm{~min}$, the media containing cellular proteins were collected. Subsequently, a $6-15 \%$ sodium dodecyl sulphate polyacrylamide gel was run under standard electrophoresis conditions, with $30 \mu \mathrm{g}$ of total protein loaded in each lane. Separated proteins were transferred to a nitrocellulose membrane (Merck KGaA) at $200 \mathrm{~mA}$ for $2 \mathrm{~h}$ and blocked with $5 \%$ non-fat milk powder at room temperature for $2 \mathrm{~h}$. Membranes with proteins were then incubated with primary antibodies (LC3, p62, p-Akt, Akt, mTOR, and p-mTOR: Rabbit polyclonal antibodies, dilution 1:1,000; GAPDH: Rabbit polyclonal antibody, dilution 1:5,000; Wuhan Sanying Biotechnology, Wuhan, China) at $4^{\circ} \mathrm{C}$ overnight. The secondary antibodies (horseradish peroxidase-conjugated secondary antibody, goat anti-rabbit, dilution 1:3,000, Wuhan Sanying Biotechnology) were subsequently added and incubated for $1 \mathrm{~h}$. Finally, all blots were developed using ECL Chemiluminescence Reagent (Merck KGaA). The levels of the target protein relative to the control GAPDH were detected by densitometric scanning using Gel-Pro Application.

Immunofluorescence analysis. Fifty cells in three independent experiments were counted. Cells were cultured on coverslips in 12 well plates at a density of $5 \times 10^{4}$ cells per well. Podocytes were washed with sterile PBS and fixed in $4 \%$ paraformaldehyde for $10 \mathrm{~min}$ at $37^{\circ} \mathrm{C}$. Afterwards, they were permeabilized with PBS containing $1 \%$ Triton X-100. After being washed twice with PBS, cells were blocked with $3 \%$ bovine serum albumin in PBS for $30 \mathrm{~min}$ and a primary antibody (p62, CD63, or LC3-II, Abcam, Cambridge, MA, USA) was added in blocking buffer overnight at a 1:200 dilution. Alexa Fluor 488-conjugated Affinipure anti-goat (1:500, Tong-Pai Bio-tech Co., Ltd.) was used as the secondary antibody. After being counterstained with 4,6-diamidino-2-phenylindole (Santa Cruz Biotechnology, Dallas, TX, USA) for $10 \mathrm{~min}$, podocytes were viewed and imaged by a laser-scanning confocal microscope (PerkinElmer, Inc., and Olympus Corporation, Waltham, MA, USA).

Apoptosis assays. Apoptosis was determined using an FITC-Annexin V/PI Apoptosis Detection Kit, according to the manufacturer instructions (Shanghai BeiBo Biotech, Shanghai, China). Cells were collected and resuspended in binding buffer. Subsequently, cells were incubated with Annexin V for $10 \mathrm{~min}$ at room temperature and $5 \mu \mathrm{l}$ of propidium iodide was added before analysis. Cells were analysed by the FAC Scan flow cytometer (BD Biosciences, Franklin Lanes, NJ, USA).

Statistical analysis. All experiments were performed a minimum of three times. SPSS 19.0 statistical software was used for the data processing. Data were compared by repeated measurement analysis of variance followed by the least significant difference post hoc test and are presented as means \pm SEM. P-value $<0.05$ was required for the results to be considered statistically significant.

\section{Results}

Podocytes exposed to the media of mesangial cells treated with aIgAl from IgAN patients showed attenuated autophagy. We measured the level of autophagy-related proteins in MPC5 cells cultured with the supernatant from MSC1097 cells treated with aIgA1 from IgAN patients. The average optical density of CD63 in the IgANs group was clearly decreased compared to the CON and the HEAs (Fig. 1, P<0.05). LC3-II binds to the autophagosomal membrane and generates puncta when the autophagosome is produced. In this study, bright puncta were clearly visible in the cytoplasm of CON group MPC5 cells, but were rare in IgAN group cells (Fig. 1). Simultaneously, p62 accumulation was observed in IgANs group podocytes. To confirm these immunofluorescence results, we evaluated LC3-II and p62 protein levels by western blotting (Fig. 2). The LC3-II/I ratio of MPC5 cells in the IgANs group decreased significantly and was only about one-third of the CON group (Fig. 2, P<0.05). In contrast, MPC5 cells in the IgANs group showed increased expression of p62 (Fig. 2, P<0.05 vs. the 

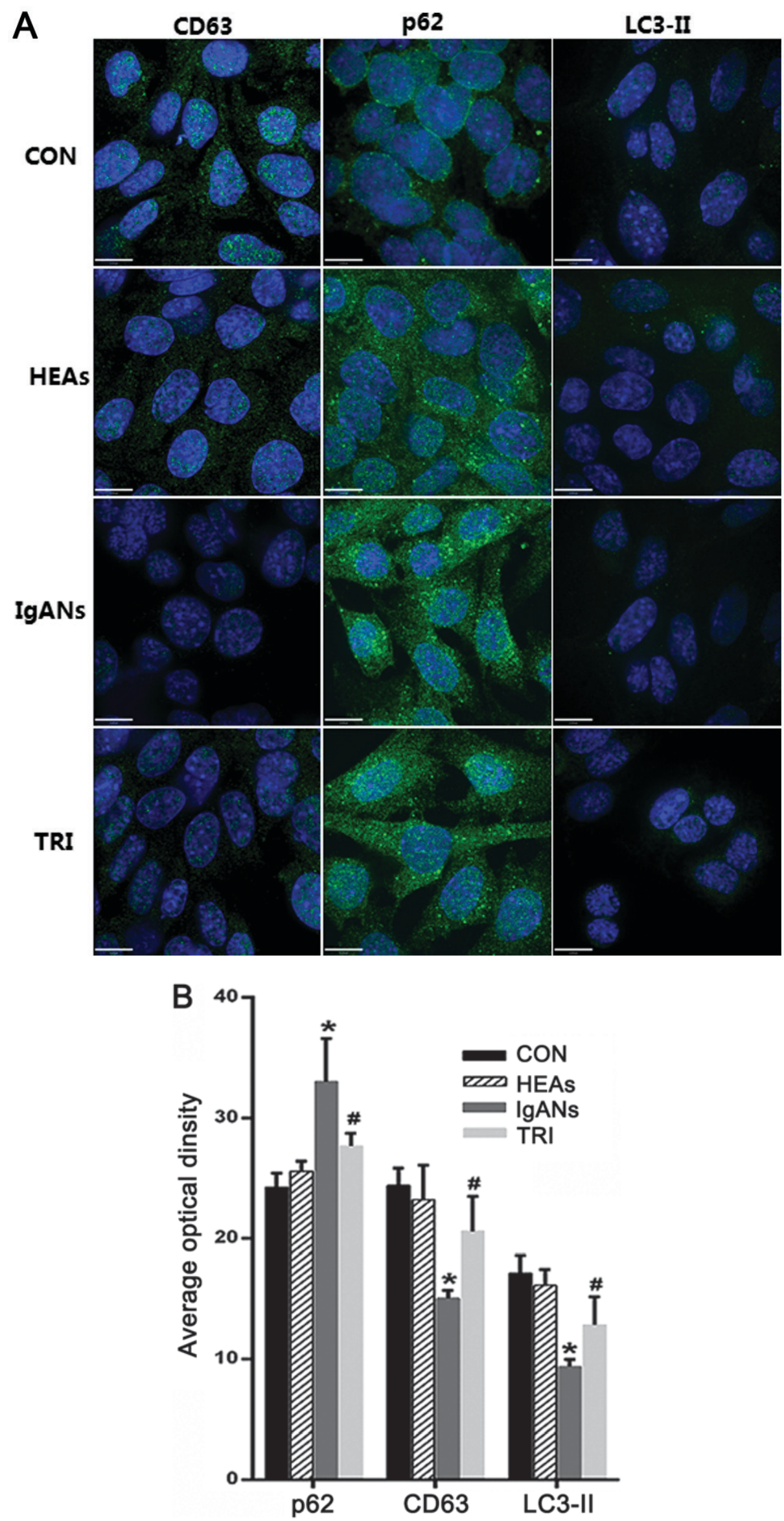

Figure 1. Immunofluorescence of autophagy markers including CD63, LC3-II and p62. (A) Immunofluorescence analysis of autophagy markers (scale bar, $13 \mu \mathrm{m}$ ). (B) Average optical density of autophagy markers in Immunofluorescence. This figure shows that autophagy was suppressed in MPC5 exposed to aIgA-MSC1097-conditioned supernatant. There were decreased levels of LC3-II and CD63 staining along with enhanced p62 in comparison with the CON and the HEAs. The situation was rescued by triptolide in the TRI group. (Magnification, $\mathrm{x} 1,000$ ). ${ }^{*} \mathrm{P}<0.05$ vs. the CON and the HEAs, ${ }^{\#} \mathrm{P}<0.05$ vs. the IgANs group. 
A

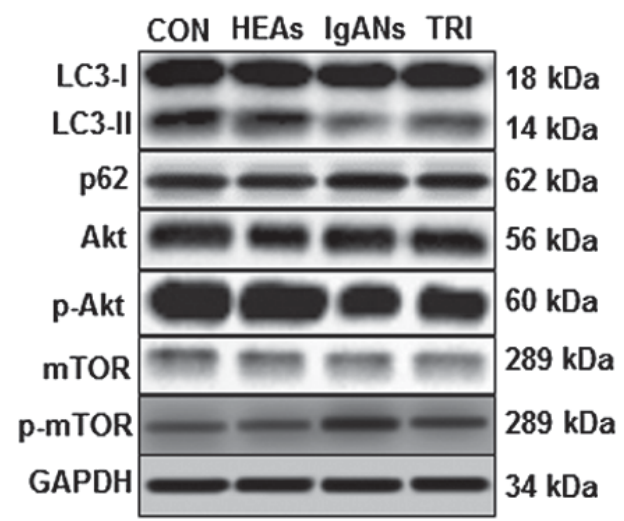

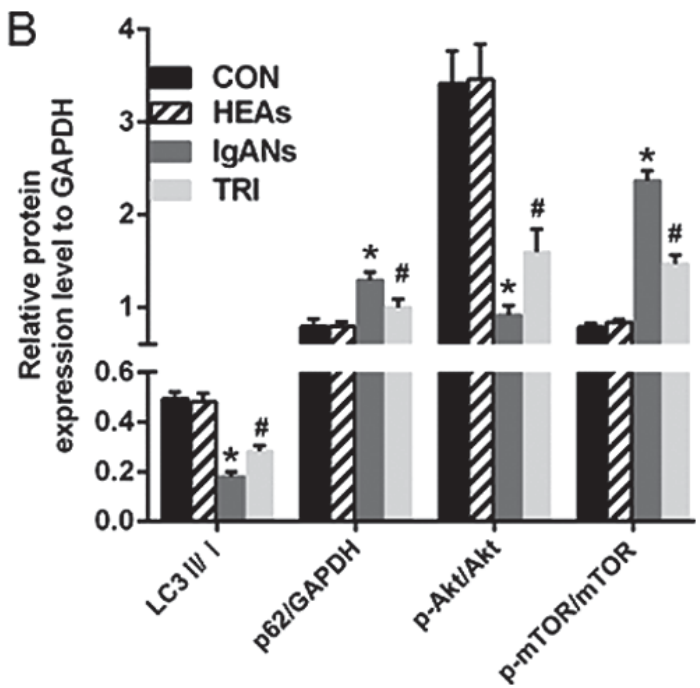

Figure 2. Western blot of autophagy-related protein of LC3, p62, p-Akt, Akt, p-mTOR, and mTOR. (A) Western blot analysis of autophagy-related proteins. (B) Densitometry of these proteins in western blots. Attenuated autophagy in podocytes was observed in the IgANs group, including a reduced LC3-II/LC3-I ratio and accumulated p62. This condition can be alleviated by triptolide in the TRI group. In addition, the ratio of p-mTOR /mTOR increased in the IgANs and decreased by triptolide in the TRI. On the contrary, the ratio of p-Akt and Akt was downregulated in podocyte from the IgANs and was upregulated in the TRI. ${ }^{*} \mathrm{P}<0.05$ vs. the CON and the HEAs, ${ }^{\#} \mathrm{P}<0.05$ vs. the group of IgANs.

CON). These western blotting results are in accordance with the immunofluorescence, and revealed attenuated autophagy in the IgANs group.

Triptolide induced podocyte autophagy potentially through $p$-mTOR/mTOR. We inferred that the PI3K/Akt pathway was triggered in IgANs group MPC5 cells with impaired autophagy. In agreement with our hypothesis, the ratio of p-mTOR/mTOR was at basal levels in the groups of CON and the HEAs, but was markedly elevated in the IgANs group (Fig. 2, P<0.001), and this was attenuated in the TRI group (Fig. 2, $\mathrm{P}<0.001$ ). Interestingly, the $\mathrm{p}-\mathrm{Akt} / \mathrm{Akt}$ ratio presented an opposite situation, where the expression of Akt was first inhibited in the IgANs group (Fig. 2, P $<0.001$ ) and then activated in the TRI group (Fig. 2, $\mathrm{P}<0.001$ ). A possible explanation is that autophagy was not mediated by the classical mTOR signalling pathway or that $\mathrm{p}$-Akt levels were modulated as a negative feedback response of p-mTOR modulation.

Triptolide induces podocyte autophagy and alleviates podocytes apoptosis. After co-culture with triptolide and the conditioned media of MPC1097 cells treated with IgAN-patient derived $\operatorname{aIg} \mathrm{A} 1$, podocytes presented a notable induction of autophagy, which was characterized by higher expression of CD63 ( $\mathrm{P}=0.039$ vs. the IgANs group, Fig. 1), obviously elevated LC3-II ( $\mathrm{P}=0.013$ vs. the IgANs group, Fig. 1), and attenuated expression of $\mathrm{p} 62(\mathrm{P}<0.01$ vs. the IgANs group). These results were also supported by western blotting, which showed a higher LC3-II/LC3-I ratio and a lower level of p62 in the TRI group (Fig. $2, \mathrm{P}<0.01$ vs. the IgANs group). We also examined the apoptosis rate of podocytes in the different groups. Fig. 3 demonstrates that the percentage of apoptotic cells increased from $9.05 \%$ in the control to $19.8 \%$ in IgANs group (Fig. 3, $\mathrm{P}<0.01)$. As expected, when compared with the IgANs group, the apoptosis rate of podocytes in TRI group exhibited obvious improvement from 19.8 to $13.2 \%$ (Fig. 3, $\mathrm{P}=0.001$ ).

\section{Discussion}

A recent study indicated that the number of patients with chronic kidney disease (CKD) in China is approximately 119 million (14), which creates a great societal burden. Glomerular disease continues to be the primary cause of CKD in China. Nowadays, IgAN is considered as the most common immune disorder related to glomerulonephritis pathogenesis (15). The single diagnostic characteristic of IgAN is the observation of aggregated IgA immune deposits in the glomerular mesangium in renal biopsy (16). Therefore, cultured mouse mesangial cells were treated with aIgA1 in this study. Moreover, aIgA1-MSC1097-conditioned medium was harvested to treat podocytes, and podocyte autophagy was subsequently evaluated in the presence or absence of triptolide.

Triptolide is among the most powerful and broadly active natural products known today (17). It is a product of the epoxidation of two terpene lactone compounds extracted from the traditional Chinese herb Tripterygium wilfordii Hook F (TWHF). Triptolide has been used in the treatment of glomerulonephritis for more than 30 years in China because of its favourable cost-benefit ratio and potent therapeutic immunosuppressive and anti-inflammatory effects (18). Having demonstrated dramatically attenuated albuminuria and renal lesions in patients with multiple kidney diseases, triptolide could be a comprehensive protective drug for delaying CKD progression. Recently, an animal study (19) demonstrated that triptolide alleviated mesangial proliferation, mesangial expansion, and glomerular IgA deposition in an IgAN rat model via anti-inflammatory effects. Triptolide can reduce proteinuria and effectively protect podocytes in rats from puromycin-induced nephropathy (20). This protective effect may be associated with the upregulation and distribution of nephrin and podocin (10). It was found that Tripterygium preparations, which are mainly composed of triptolide, could ameliorate proteinuria due to the enhanced expression 

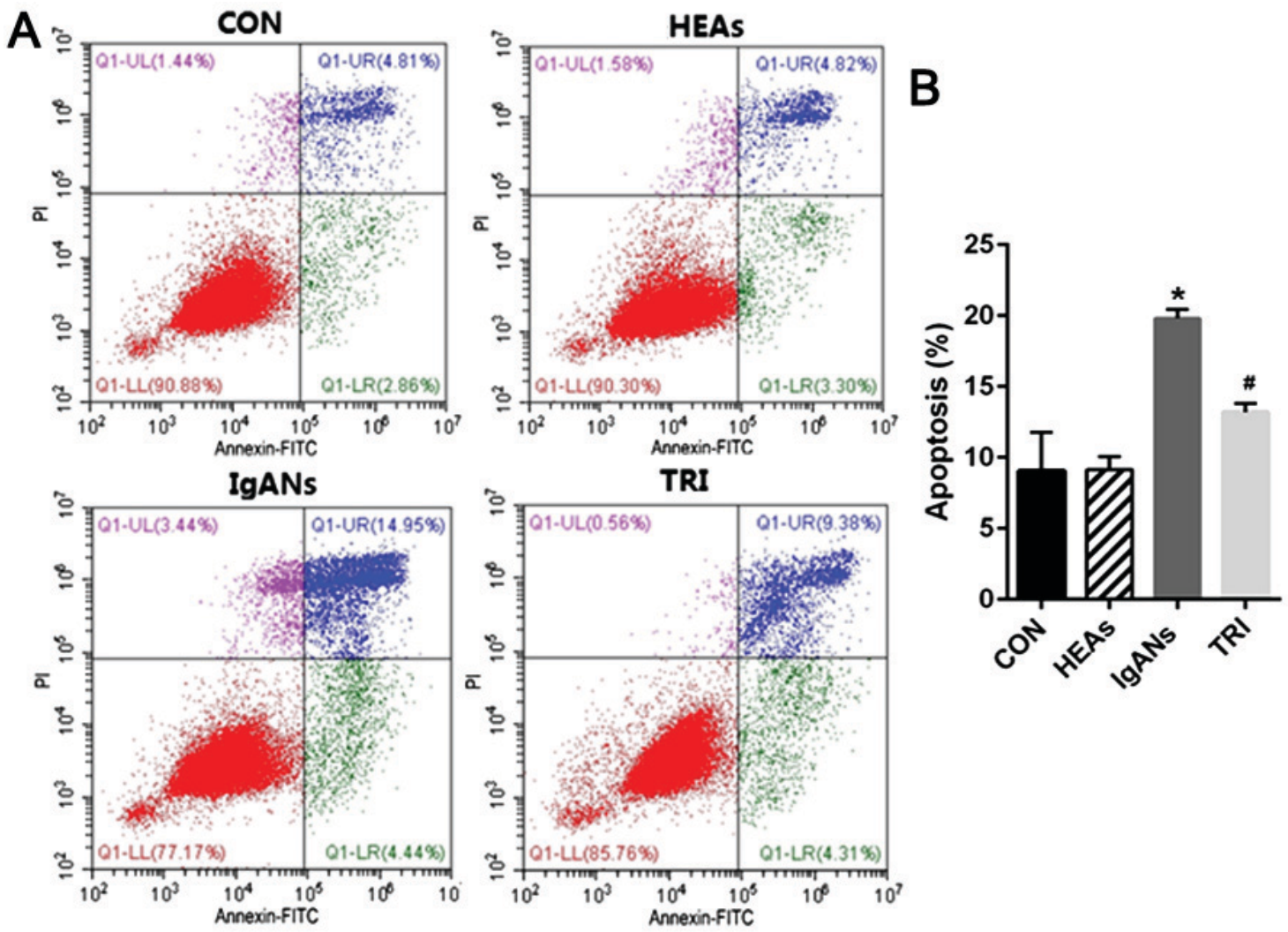

Figure 3. Apoptosis measurement of apoptosis by flow cytometry. (A) Flow cytometry Annexin V/PI staining for measuring apoptosis after 24 h exposure of MPC-5 cells to the aIgA-MSC1097-conditioned supernatant. (B) The corresponding linear diagram of flow cytometry. The IgANs presented a significant increase in the rate of podocytes apoptosis when compared to the CON and the HEAs. Triptolide reduced the apoptosis of MPC5 cells. * $<<0.05$ vs. the CON and the HEAs, ${ }^{\#} \mathrm{P}<0.05$ vs. the IgANs.

of nephrin (21). Triptolide has been reported to markedly attenuate albuminuria and improve podocyte injury in a rat model of diabetic nephropathy, possibly due to its inhibitory effects on inflammation and macrophage infiltration in the kidney (22). In addition, triptolide may attenuate podocyte injury in rats with adriamycin-induced nephropathy by regulating expression of miRNA-344b-3p and miRNA-30b-3p (23). These data showed that triptolide can protect podocytes from from different kinds of injury. Autophagy is very important for the maintenance of end-differentiated cells such as podocytes. Therefore, we inferred that the protective effects of triptolide on podocytes can be correlated to autophagy and conducted this study to observe whether triptolide can maintain podocyte autophagy capability.

It has been reported that podocyte autophagy is involved in the development of $\operatorname{IgAN}$. Two types of autophagy were found (24) in podocytes obtained from renal biopsies of 16 children. Type I rarely transformed to autophagic vacuoles and did not dissolve, while Type II frequently transformed to autophagosomes and autophagic vacuoles, thus facilitating protein and lipid clearance. Therefore, it was concluded that type I autophagy was correlated with histopathologically more progressive disease, possibly reflecting a tendency towards poorer prognosis. We have previously reported (25) that podocytes from the renal biopsies of $\operatorname{Ig} \mathrm{AN}$ patients possessed more autophagosomes than healthy subjects did. In this study, we found that autophagy was reduced in podocytes exposed to IgAN-derived aIgA1-conditioned medium compared to the
CON group. This autophagy dysfunction was characterized by decreased LC3-II and increased p62 levels. Collectively, this evidence indicated that podocyte autophagy was suppressed during the progression of $\operatorname{IgAN}$. In this study, we found that autophagy inhibition was recovered when podocytes were exposed to triptolide. The results indicated that triptolide can induce podocyte autophagy, promote the conversion of LC3-I to LC3-II, as well as the elimination of p62. Similar phenomena have been reported in other cell types. In an animal study it was found that the administration of triptolide $12 \mathrm{~h}$ prior to middle cerebral artery occlusion reduced infarction area through the activation of autophagy (26). Triptolide could induce autophagy and promote the clearance of both WT and A53T $\alpha$-synuclein in neurons (27).

Another finding of this study was that podocyte autophagy in IgAN was suppressed or induced by triptolide through p-mTOR/mTOR. It is well known that the PI3K/Akt pathway is one of the most important signalling pathways regulating autophagy. Phosphorylated Akt can promote the formation of p-mTOR, an inhibitor of cell autophagy. The activation of the PI3K/Akt pathway was the main cause of impaired autophagy in ELK3-knockdown MDA-MB-231 cells (28). Surprisingly, we found increased p-mTOR and decreased p-Akt in the IgANs group podocytes, whereas we detected decreased p-mTOR and elevated p-Akt in the TRI group podocytes. An alternative hypothesis may be that other signalling pathways are involved. Another possible reason could be that the modulation of $\mathrm{p}$-Akt was a response to the modulation of $\mathrm{p}-\mathrm{mTOR}$. 


\section{Acknowledgements}

Not applicable.

\section{Funding}

The present study was funded by the Project of Scientific research Foundation of Chinese Medicine (grant no. 2014ZB002).

\section{Availability of data and materials}

The datasets used and/or analysed during the current study are available from the corresponding author on reasonable request.

\section{Authors' contributions}

SL and QH designed the concept and performed the experiments. JJ and XS performed the experiments. SL and XJ analysed and interpreted the data. YL was involved in analyzing data and drafting the manuscript. All authors read and approved the final manuscript.

\section{Ethics approval and consent to participate}

All procedures performed in studies involving human participants were in accordance with the ethical standards of the institutional and/or national research committee. The study has obtained human research ethics approval from the local ethics committee and all subjects provided informed consent (approval no. KY2014011).

\section{Patient consent for publication}

Informed consent was obtained from all individual participants included in the study.

\section{Competing interests}

The authors declare that they have no competing interests.

\section{References}

1. Berger $\mathrm{J}$ and Hinglais $\mathrm{N}$ : Intercapillary deposits of $\operatorname{IgA}-\operatorname{IgG}$ J Urol Nephrol (Paris) 74: 694-695, 1968 (In French).

2. Gharavi AG, Kiryluk K, Choi M, Li Y, Hou P, Xie J, Sanna-Cherchi S, Men CJ, Julian BA, Wyatt RJ, et al: Genome-wide association study identifies susceptibility loci for IgA nephropathy. Nat Genet 43: 321-327, 2011.

3. Wang C, Tang Y, Peng H, Ye ZC, Chen ZJ, Yu XQ and Lou TQ Supernatant of cultured mesangial cells with $\operatorname{IgA} 1$ from $\operatorname{Ig} \mathrm{A}$ nephropathy induces apoptosis of podocyte. Chin J Nephrol 24: 387-391, 2008

4. Wang C, Ye Z, Peng H, Tang H, Liu X, Chen Z, Yu X and Lou T: Effect of aggregated immunoglobulin A1 from immunoglobulin A nephropathy patients on nephrin expression in podocytes. Nephrology (Carlton) 14: 213-218, 2009.

5. Zhu L, Zhang Q, Shi S,Liu L, Lv J and Zhang H: Synergistic effect of mesangial cell-induced CXCL1 and TGF- $\beta 1$ in promoting podocyte loss in IgA nephropathy. PLoS One 8: e73425, 2013.

6. Tewari R, Nada R, Rayat CS, Boruah D, Dudeja P, Joshi K and Sakhuja V: Correlation of proteinuria with podocyte foot process effacement in IgA nephropathy: An ultrastructural study. Ultrastruct Pathol 39: 147-151, 2015.

7. Shen P, Shen J, Li W and He L: Urinary podocyte can be an indicator for the pathogenetic condition of patients with $\operatorname{IgA}$ nephropathy. Clin Lab 60: 1709-1715, 2014.
8. Yasuda-Yamahara M, Kume S, Tagawa A, Maegawa $\mathrm{H}$ and Uzu T: Emerging role of podocyte autophagy in the progression of diabetic nephropathy. Autophagy 11: 2385-2386, 2015.

9. Hartleben B, Gödel M, Meyer-Schwesinger C, Liu S, Ulrich T, Köbler S, Wiech T, Grahammer F, Arnold SJ, Lindenmeyer MT, et al: Autophagy influences glomerular disease susceptibility and maintains podocyte homeostasis in aging mice. J Clin Invest 120: 1084-1096, 2010.

10. Li XJ, Jiang ZZ and Zhang LY: Triptolide: Progress on research in pharmacodynamics and toxicology. J Ethnopharmacol 155: $67-79,2014$

11. Liang S, Jin J, Lin B, Gong J, Li Y and He Q: Rapamycin induces autophagy and reduces the apoptosis of podocytes under a stimulated condition of immunoglobulin A nephropathy. Kidney Blood Press Res 42: 177-187, 2017.

12. Dai Q, Liu J, Du YL, Hao X, Ying J, Tan Y, He LQ, Wang WM and Chen N: Histone deacetylase inhibitors attenuate $\mathrm{P}-\mathrm{aIgA}$ 1-induced cell proliferation and extracellular matrix synthesis in human renal mesangial cells in vitro. Acta Pharmacol Sin 37: 228-234, 2016

13. Yang Q, Sun M, Chen Y, Lu Y, Ye Y, Song H, Xu X, Shi S and Wang J: Triptolide protects podocytes from TGF- $\beta$-induced injury by preventing miR-30 downregulation. Am J Transl Res 9: $5150-5159,2017$

14. Liu ZH: Nephrology in China. Nat Rev Nephrol 9: 523-528, 2013.

15. Wyatt RJ and Julian BA: IgA nephropathy. N Engl J Med 368: 2402-2414, 2013.

16. Lin M, Du L, Brandtzaeg P and Pan-Hammarström Q: IgA subclass switch recombination in human mucosal and systemic immune compartments. Mucosal Immunol 7: 511-520, 2014.

17. Graziose R, Lila MA and Raskin I: Merging traditional Chinese medicine with modern drug discovery technologies to find novel drugs and functional foods. Curr Drug Discov Technol 7: 2-12,2010.

18. Liu Q: Triptolide and its expanding multiple pharmacological functions. Int Immunopharmacol 11: 377-383, 2011.

19. He L, Peng X, Liu G, Tang C, Liu H, Liu F, Zhou H and Peng Y: Anti-inflammatory effects of triptolide on IgA nephropathy in rats. Immunopharmacol Immunotoxicol 37: 421-427, 2015.

20. Zheng CX, Chen ZH, Zeng CH, Qin WS, Li LS and Liu ZH: Triptolide protects podocytes from puromycin aminonucleoside induced injury in vivo and in vitro. Kidney Int 74: 596-612, 2008.

21. Wang YG, Sun W and Zhen YJ: Preventive effect of multi-glycoside of Tripterygium wilfordii Hook. f. on proteinuria and mesangial injury in experimental mesangial proliferative glomerulonephritis. Zhongguo Zhong Xi Yi Jie He Za Zhi 25: 817-821, 2005 (In Chinese).

22. Ma R, Liu L, Liu X, Wang Y, Jiang W and Xu L: Triptolide markedly attenuates albuminuria and podocyte injury in an animal model of diabetic nephropathy. Exp Ther Med 6: 649-656, 2013.

23. Jiang CB, Wei MG, Tu Y, Zhu H, Li CQ, Jing WM and Sun W: Triptolide attenuates podocyte injury by regulating expression of miRNA-344b-3p and miRNA-30b-3p in rats with adriamycin-induced nephropathy. Evid Based Complement Alternat Med 2015: 107814, 2015.

24. Sato S, Yanagihara T, Ghazizadeh M, Ishizaki M, Adachi A, Sasaki Y, Igarashi T and Fukunaga Y: Correlation of autophagy type in podocytes with histopathological diagnosis of IgA nephropathy. Pathobiology 76: 221-226, 2009.

25. Liang S, Jin J, Gong J, Lin B, Li Y and He Q: How many podocyte autophagosomes are there in immunoglobulin A nephropathy and idiopathic membranous nephropathy? Int Urol Nephrol 48: 2109-2114, 2016.

26. Yang Y, Gao K, Hu Z, Li W, Davies H, Ling S, Rudd JA and Fang M: Autophagy upregulation and apoptosis downregulation in DAHP and triptolide treated cerebral ischemia. Mediators Inflamm 2015: 120198, 2015

27. Hu G, Gong X, Wang L, Liu M, Liu Y, Fu X, Wang W, Zhang T and Wang X: Triptolide promotes the clearance of $\alpha$-synuclein by enhancing autophagy in neuronal cells. Mol Neurobiol 54: 2361-2372, 2017

28. Park JH, Kim KP, Ko JJ and Park KS: PI3K/Akt/mTOR activation by suppression of ELK3 mediates chemosensitivity of MDA-MB-231 cells to doxorubicin by inhibiting autophagy. Biochem Biophys Res Commun 477: 277-282, 2016.

This work is licensed under a Creative Commons Attribution-NonCommercial-NoDerivatives 4.0 International (CC BY-NC-ND 4.0) License. 